\title{
Influência da utilização de procedimentos não padronizados de ensaio para a determinação experimental da resistência à compressão simples e do módulo estático de elasticidade do cimento Portland
}

\author{
Influence of non standard test procedures for \\ determination of compressive strength and \\ static modulus of elasticity of Portland cement
}

\author{
Jacinto Manuel Antunes de Almeida ${ }^{1}$, Bruno do Vale Silva ${ }^{1}$, \\ Josué Argenta Chies ${ }^{1}$, Josiane Gasperin ${ }^{1}$, \\ Luiz Carlos Pinto da Silva Filho ${ }^{1}$.
}

\footnotetext{
${ }^{1}$ Laboratório de Ensaios e Modelos Estruturais - LEME (http://www.ufrgs.br/leme). Universidade Federal do Rio Grande do Sul - UFRGS. Av. Bento Gonçalves, n. 9500 - Prédio 43436, Porto Alegre, RS

e-mail: jass_carnival@hotmail.com; dovalesilva@hotmail.com; jogasperin@ibest.com.br; josuchies@hotmail.com; lcarlos66@gmail.com.
}

\begin{abstract}
RESUMO
A resistência à compressão é um dos parâmetros fundamentais usados para controle e caracterização do cimento. O ensaio padrão para determinação da resistência à compressão simples do cimento Portland, normatizado pela NBR 7215 [1]], especifica o uso de areia com granulometria determinada e estabelece procedimentos de mistura rigorosos na preparação dos corpos de prova. Sabe-se que, nem sempre, os procedimentos padrão são rigorosamente seguidos, seja devido a fatores relacionados com competência técnica ou pela simplificação da norma, entre outros fatores. O objetivo principal deste trabalho foi avaliar a influência de determinadas simplificações dos procedimentos padrão de ensaio na determinação experimental da resistência à compressão simples do cimento Portland. Foram testados diferentes procedimentos de mistura e utilizados diferentes tipos de agregado miúdo, especificamente, areia média comum ou com granulometria controlada, tomando como referência a denominada areia do Tietê. Foram utilizadas duas amostras de cimento CPV-ARI, provenientes de fabricantes distintos. Para todas as combinações foram realizados ensaios de resistência à compressão e módulo estático de elasticidade nas idades de 1, 3, 7 e 28 dias. Foi efetuada uma análise de variância (ANOVA) para avaliar se as variáveis estudadas geravam diferenças estatisticamente significativas nos resultados experimentais. Foi possível concluir que, de forma geral, a moldagem simplificada não influenciou significativamente a resistência à compressão simples nem o módulo estático de elasticidade do cimento Portland. Por outro lado, o tipo de agregado miúdo utilizado influenciou significativamente a resistência à compressão, mas não impactou o módulo de elasticidade das amostras.
\end{abstract}

Palavras-chave: Cimento Portland, propriedades mecânicas, ensaios padronizados, ensaios não padronizados.

\begin{abstract}
The compressive strength is one of the basic parameters to control and characterization of cement. According to the NBR 7215 [1], the standard test procedure for determination of compressive strength of cement specifies the use of sand with a specific gradation and establishes specific rules for the preparation of the standard mixture of mortar, used to manufacture the test specimens. It is known that these recommendations are not always followed when testing. In the context of the present study, we aimed to investigate the influence of some deviations from the standard procedure in the results of compressive strength and modulus of elasticity of mortar. We've tested a simplified mixing procedure and the use of average sand or with controlled gradation, taking as reference the so-called sand from Tietê. We've also used two samples of CPV-ARI cement from different manufacturers. For all combinations, tests were performed to determine the compressive
\end{abstract}


strength and static modulus of elasticity of mortar at 1, 3, 7 and 28 days of age. An analysis of variance (ANOVA) was performed to assess whether the variables generated significant differences. The results show that the simplified mixing procedure didn't affect the compressive strength neither the modulus of elasticity of Portland cement. The different types of sand tested only affected the compressive strength of Portland cement.

Keywords: Portland cement, mechanical properties, standard testing; non standard testing.

\section{INTRODUÇÃO}

No Brasil, a demanda por cimento tem crescido consideravelmente nos últimos anos, acompanhando a expansão da indústria da construção civil. Para NEVILLE [2], o sucesso do cimento Portland se deve, em parte, ao fato de ser um pó com excelentes propriedades adesivas e coesivas, capaz de ligar fragmentos de minerais entre si de modo a formar um todo compacto. $\mathrm{O}$ desempenho do cimento pode ser influenciado por sua composição química e também por suas propriedades físicas como, por exemplo, sua finura. MEHTA \& MONTEIRO [3] creditam a formação das propriedades mecânicas a reações químicas que ocorrem com a hidratação do cimento e posterior endurecimento da argamassa. Ainda de acordo com os autores, essas reações ocorrem quando os compostos do cimento $\left(\mathrm{C}_{4} \mathrm{AF}, \mathrm{C}_{3} \mathrm{~S}, \mathrm{C}_{2} \mathrm{~S}\right.$ e $\left.\mathrm{C}_{3} \mathrm{~A}\right)$ reagem com a água formando o hidróxido de cálcio $\left(\mathrm{Ca}(\mathrm{OH})_{2}\right)$ e o silicato de cálcio hidratado (C-S-H) que, por se tratar de um composto bastante resistente, torna-se o principal responsável pelas propriedades mecânicas do material.

De acordo com CARNEIRO [4], a areia é a fração inerte que compõe a argamassa e, embora não participe das reações químicas de endurecimento, sua inserção interfere tanto no estado fresco da argamassa, quanto no estado endurecido. Segundo o autor, a composição granulométrica e o formato dos grãos interferem, diretamente, no estado fresco, na trabalhabilidade e na retenção de água e, no estado endurecido, nas resistências mecânicas, na capacidade de deformação e na permeabilidade, isto em função do preenchimento dos vazios e também pela dinâmica de rolamento entre partículas. Da mesma forma, ARAÚJO [్] afirma que a forma e a textura superficial da areia influenciam de forma significativa as propriedades das argamassas, tanto no estado fresco, quanto no endurecido, e acrescenta, também, a influência das mesmas durante o período de uso. Para SCHUTTER E POPPE [6], o tipo de areia influencia significativamente os parâmetros reológicos e propriedades mecânicas da argamassa, sendo seu estudo importante na dosagem.

No Brasil, a determinação experimental da resistência à compressão do cimento Portland deve ser realizada de acordo com a NBR 7215 [1], que estabelece os materiais e equipamentos de ensaio, assim como, os procedimentos de mistura para a fabricação da argamassa utilizada na moldagem das amostras. Complementarmente, a NBR 7214 [7] especifica a areia para ensaio e a NBR 5733 [8] regula as condições exigíveis no recebimento do CP V-ARI, sejam condições gerais, entre as quais, composição e armazenamento em sacos, ou condições específicas que o material deve atender, nomeadamente, exigências químicas, físicas e mecânicas.

Qualquer falha operacional, seja na moldagem ou nos procedimentos de ensaio, pode influenciar a resistência do material, advindo desse conceito a importância de executar corretamente as recomendações padronizadas de ensaio. Habitualmente, os vários fatores que podem condicionar o rigor dos ensaios realizados em laboratório estão associados a dificuldades operacionais ou à falta de laboratoristas certificados pelo INMETRO / IBRACON (fatores relativos à competência técnica) [9]. Além disso, outros fatores podem contribuir significativamente para a variação dos resultados experimentais, mas, habitualmente, não são quantificados, entre os quais: equipamento inadequado ou calibração incorreta do equipamento de ensaio, acondicionamento inadequado dos materiais, utilização de materiais alternativos, caracterização incorreta ou incompleta dos materiais, preparação incorreta das amostras, procedimentos de ensaio modificados, simplificados ou etapas eliminadas e imprevistos materiais e humanos.

O objetivo principal deste trabalho foi avaliar a influência de determinadas simplificações das recomendações da NBR 7215 [1] na determinação experimental da resistência à compressão simples do cimento Portland. Desta forma, procurou-se avaliar se seria aceitável a utilização de areias alternativas e procedimentos simplificados de mistura, sem comprometer os resultados da resistência à compressão simples e do módulo estático de elasticidade das amostras. Numa fase complementar, foram testados os modelos teóricos da NBR 6118 [10] e do CEB 90 [11] utilizados no cálculo do módulo estático de elasticidade.

\section{MATERIAIS E MÉTODOS}

Neste item é apresentado o programa experimental deste trabalho com a descrição das principais características dos materiais e métodos utilizados. 


\subsection{Matriz experimental}

Os procedimentos recomendados pela NBR 7215 [1] são rigorosos nas etapas e intervalos de tempo definidos para a mistura mecânica dos materiais. Com o intuito de avaliar a influência da competência técnica foram testados dois procedimentos de preparação das amostras para ensaio: a) procedimento padrão e b) procedimento simplificado de mistura, não padronizado, definido pela equipe de pesquisa, com tempos e ordem de mistura dos materiais de acordo com o descrito no item 2.3.

Para avaliar a influência da utilização de areias alternativas na confecção das amostras, devido ao custo e à escassez da areia padrão para ensaio, foram testados três tipos de agregado miúdo: areia padronizada de acordo com a NBR 7214 [7]; areia média natural com granulometria contínua e sem fracionamento prévio; areia média natural com fracionamento padronizado realizado em laboratório.

As variáveis escolhidas foram combinadas de acordo com o procedimento de mistura (Moldagem A e Moldagem B) e tipo de agregado miúdo utilizado (Agregado I, Agregado II e Agregado III) para duas amostras do mesmo tipo de cimento (CPV-ARI proveniente de dois fabricantes distintos: Fabricante 1 e Fabricante 2). A Tabela 1 resume a matriz experimental do estudo. No total, foram moldados e ensaiados 72 corpos de prova cilíndricos de argamassa de cimento Portland, com dimensões 50x100 mm.

Tabela 1: Resumo da matriz experimental.

\begin{tabular}{ll}
\hline VARIÁVEIS & DESIGNAÇÃo \\
\hline Moldagem (mistura) & A / B \\
Agregado (areia) & I / II / III \\
Idade (dias) & 1 / 3 / 7 / 28 \\
\hline 72 CPs cilíndricos 50x100 mm \\
\hline \multicolumn{2}{l}{ Mistura padrão: Fabricante 1 + Moldagem A + Agregado I } \\
\hline
\end{tabular}

\subsection{Materiais}

Neste trabalho, foram utilizadas duas amostras de cimento CPV-ARI, oriundas de diferentes fabricantes (Fabricante 1 e Fabricante 2), com composições físicas e químicas diferenciadas. As Tabelas 2 e 3 apresentam resumidamente a caracterização física e química dos cimentos empregados.

Tabela 2: Caracterização física e química do cimento do Fabricante 1.

\begin{tabular}{|c|c|c|c|}
\hline CARACTERÍSTICAS & $\begin{array}{l}\text { ANÁLISE DO } \\
\text { CIMENTO }\end{array}$ & $\begin{array}{l}\text { EXIGÊNCIA } \\
\text { DA NORMA }\end{array}$ & NBR \\
\hline Área específica Blaine $\left(\mathrm{cm}^{2} / \mathrm{g}\right)$ & 4401 & $\geq 3000$ & NM 76/98 \\
\hline Tempo de pega & & & NM 65/02 \\
\hline Início (min) & 140 & $\geq 60$ & \\
\hline Término (min) & 187 & $\leq 600$ & \\
\hline Massa Específica (g/cm³) & 3,11 & - & NM 23/01 \\
\hline Diâmetro médio $(\mu \mathrm{m})$ & 11,06 & - & \\
\hline Perda ao Fogo (\%) & 2,56 & $\leq 4,5$ & NM 18/04 \\
\hline RI (\%) & 0,38 & $\leq 1,0$ & NM 15/04 \\
\hline MgO (\%) & 1,49 & $\leq 6,5$ & NM 21/04 \\
\hline $\mathrm{SO}_{3}(\%)$ & 3,02 & $\leq 4,5$ & NM 16/09 \\
\hline $\mathrm{SiO}_{2}(\%)$ & 17,83 & - & \\
\hline $\mathrm{CaO}(\%)$ & 68,11 & - & \\
\hline
\end{tabular}


Tabela 3: Caracterização física e química do cimento do Fabricante 2.

\begin{tabular}{|c|c|c|c|}
\hline CARACTERÍSTICAS & $\begin{array}{l}\text { ANÁLISE DO } \\
\text { CIMENTO }\end{array}$ & $\begin{array}{l}\text { EXIGÊNCIA } \\
\text { DA NORMA }\end{array}$ & NBR \\
\hline Área específica Blaine (cm²/g) & 4298 & $\geq 3000$ & NM 76/98 \\
\hline Tempo de pega & & & NM 65/02 \\
\hline Início (min) & 160 & $\geq 60$ & \\
\hline Término (min) & 270 & $\leq 600$ & \\
\hline Massa Específica (g/cm³) & 2,96 & - & NM 23/01 \\
\hline Diâmetro médio $(\mu \mathrm{m})$ & 8,40 & & \\
\hline Perda ao Fogo (\%) & 3,03 & $\leq 4,5$ & NM 18/04 \\
\hline RI (\%) & 0,20 & $\leq 1,0$ & NM 15/04 \\
\hline MgO (\%) & 1,74 & $\leq 6,5$ & NM 21/04 \\
\hline $\mathrm{SO}_{3}(\%)$ & 3,11 & $\leq 4,5$ & NM 16/09 \\
\hline $\mathrm{SiO}_{2}(\%)$ & 19,84 & - & \\
\hline $\mathrm{CaO}(\%)$ & 64,03 & - & \\
\hline
\end{tabular}

Para confecção das argamassas, utilizou-se como agregado miúdo uma areia com granulometria padronizada de acordo com a norma (Agregado I), uma areia quartzosa natural com distribuição granulométrica contínua (Agregado II) e a mesma areia natural do Agregado II distribuída em 4 frações padronizadas idênticas ao Agregado I (Agregado III). Os agregados I, II e III são caracterizados quanto as suas propriedades físicas, de acordo com as Tabelas 4, 5 e 6, respectivamente.

Tabela 4: Caracterização física do agregado miúdo padronizado - Agregado I.

\begin{tabular}{lll}
\hline ABERTURA DA & \% & \% RETIDA \\
PENEIRA (mm) & RETIDA & ACUMULADA \\
\hline 2,36 & 0,00 & 0,00 \\
1,18 & 22,00 & 26,00 \\
0,60 & 23,00 & 49,00 \\
0,30 & 24,00 & 73,00 \\
0,15 & 25,00 & 98,00 \\
Fundo & 2,00 & 100 \\
\hline Dimensão máxima característica $=2,36 \mathrm{~mm}$ & \\
Módulo de finura = 2,46 & \\
Massa específica = 2,64 g/cm ${ }^{3}$ & \\
Umidade = 0,0\% & \\
\hline
\end{tabular}


Tabela 5: Caracterização física do agregado miúdo natural - Agregado II.

\begin{tabular}{lll}
\hline $\begin{array}{l}\text { ABERTURA DA } \\
\text { PENEIRA (mm) }\end{array}$ & \% RETIDA & $\begin{array}{l}\text { \% RETIDA } \\
\text { ACUMULADA }\end{array}$ \\
\hline 6,30 & 0,00 & 0,00 \\
4,75 & 0,15 & 0,15 \\
2,36 & 3,84 & 3,99 \\
1,18 & 9,96 & 13,94 \\
0,60 & 29,88 & 43,82 \\
0,30 & 45,25 & 89,07 \\
0,15 & 0,13 & 89,21 \\
Fundo & 10,79 & 100 \\
\hline Dimensão máxima característica $=2,36 \mathrm{~mm}$ & \\
Módulo de finura = 2,40 & \\
Massa específica $=2,52 \mathrm{~g} / \mathrm{cm}^{3}$ & \\
Umidade = 0,3\% & \\
\hline
\end{tabular}

Tabela 6: Caracterização física do agregado miúdo natural padronizado - Agregado III.

\begin{tabular}{lll}
\hline ABERTURA DA & \% RETIDA & $\begin{array}{l}\text { \% RETIDA } \\
\text { ACUMULADA }\end{array}$ \\
PENEIRA (mm) & & 0,00 \\
2,36 & 0,00 & 25,00 \\
1,18 & 25,00 & 50,00 \\
0,60 & 25,00 & 75,00 \\
0,30 & 25,00 & 100 \\
0,15 & 25,00 & 100 \\
Fundo & 0,00 & \\
Dimensão máxima característica $=2,36 \mathrm{~mm}$ & \\
Módulo de finura = 2,50 & \\
Massa específica = 2,52 g/cm & \\
Umidade $=0,3 \%$ & \\
\hline
\end{tabular}

\subsection{Metodologia}

A confecção da argamassa de cimento foi realizada em misturador mecânico de argamassa de acordo com as especificações da norma, na temperatura ambiente de $(23 \pm 2)^{\circ} \mathrm{C}$. As quantidades de material utilizado permitiram a moldagem de 6 corpos de prova cilíndricos de 50x100 mm em cada argamassada. Foram testados dois procedimentos de mistura: procedimento de acordo com a NBR 7215 [1] (Moldagem A) e procedimento simplificado, desenvolvido pela equipe de pesquisa (Moldagem B).

A Moldagem A foi executada, de acordo com a norma brasileira, da seguinte forma:

- Primeiramente adicionou-se na cuba toda a quantidade de água, de $300 \pm 0,2$ g; em seguida, acrescentou-se $624 \pm 0,4 \mathrm{~g}$ de cimento Portland CP V-ARI; a mistura do cimento foi feita com o misturador na velocidade baixa, durante 30 segundos;

- Após este tempo, e sem paralisar a operação de mistura, iniciou-se a colocação da areia padronizada, com quatro frações de $25 \%$ de cada granulometria estipulada em norma (ou seja, as retidas na peneira 1,2; 0,6; 0,3 e 0,15), cada fração com $468 \pm 0,3 \mathrm{~g}$; a areia foi colocada gradualmente, na velocidade baixa, durante o tempo de 30 segundos;

- Imediatamente após o término da colocação da areia, mudou-se para a velocidade alta, misturando-se os materiais nesta velocidade durante 30 segundos; 
- Após este tempo, desligou-se o misturador durante 1 minuto e 30 segundos, sendo que nos primeiros 15 segundos retirou-se, com auxílio de uma espátula, a argamassa que ficou aderida às paredes da cuba e à pá colocando-a novamente no interior da cuba; durante o tempo restante, 1 minuto e 15 segundos, a argamassa ficou em repouso na cuba, coberta com pano limpo e úmido;

- Logo após este intervalo, para finalizar a mistura, ligou-se o misturador na velocidade alta, por mais 1 minuto. Foi registrada a hora em que o cimento foi colocado em contato com a água da mistura;

- Por fim, os corpos de prova foram moldados em quatro camadas, com adensamento manual, dandose 30 golpes em cada camada com auxílio de um soquete.

A Moldagem B foi realizada com as mesmas quantidades de material, de acordo com os seguintes procedimentos:

- Primeiramente se colocou na cuba toda a quantidade de cimento e areia e misturaram-se estes materiais em velocidade baixa por 30 segundos;

- Em seguida, e sem paralisar a operação, se adicionou toda a quantidade de água durante um período de tempo de 30 segundos, ainda em velocidade baixa;

- Imediatamente após o término da colocação da água, mudou-se para a velocidade alta, misturando-se os materiais por 1 minuto;

- Depois desse tempo, desligou-se o misturador durante 1 minuto e 30 segundos; durante este período, retirou-se, com auxilio de uma espátula, a argamassa que ficou aderida às paredes da cuba e a pá do misturador;

- Logo após o período de repouso, ligou-se o misturador por mais 1 minuto em velocidade alta;

- Por fim, os corpos de prova foram moldados, em duas camadas uniformes, com auxílio de mesa vibratória.

Foram desmoldados os corpos de prova 24 horas após a moldagem e colocados em cura submersa em solução saturada de hidróxido de cálcio com temperatura de $23 \pm 2^{\circ} \mathrm{C}$ até duas horas antes dos ensaios, com exceção das amostras ensaiadas com 24 horas de idade. Na data da ruptura, os corpos de prova foram retificados para regularização das superfícies.

Para cada combinação estudada, foram utilizados três corpos de prova cilíndricos de 50x100 mm por idade. Optou-se por reduzir o número de corpos de prova de seis, número recomendado pela norma brasileira, para três de forma a reduzir o programa experimental sem comprometer a qualidade dos resultados. As amostras foram submetidas ao ensaio de resistência à compressão simples e de módulo estático de elasticidade, para as idades de 1, 3, 7 e 28 dias. Os ensaios foram realizados no Laboratório de Ensaios e Modelos Estruturais (LEME/UFRGS). Inicialmente, os corpos de prova foram instrumentados com dois transdutores de deslocamento do tipo Linear Variable Differential Transformer (LVDT) e, posteriormente, todo o conjunto foi colocado diretamente sob o prato inferior da prensa, modelo Shimadzu UH-I, centrado em relação ao eixo de carregamento. Foi aplicada carga de compressão à velocidade constante de $0,25 \pm 0,05 \mathrm{MPa} / \mathrm{s}$ até à ruptura, e as medições, quer da carga, quer da deformação específica, foram registradas no software Trapezium 2.

\section{RESULTADOS}

Neste item são apresentados os resultados dos ensaios, para cada combinação de variáveis estudadas. A Tabela 7 resume os valores experimentais obtidos para a resistência média à compressão do cimento Portland, $\mathrm{f}_{\mathrm{cm}}$, e o desvio padrão, para cada uma das variáveis estudadas. 
Tabela 7: Valores médios para a resistência à compressão simples do cimento Portland.

\begin{tabular}{lllllll}
\hline & \multicolumn{3}{c}{ Fabricante 1 } & \multicolumn{2}{c}{ Fabricante 2 } \\
\cline { 2 - 7 } & Moldagem A & Moldagem B & Moldagem A & Moldagem A & Moldagem A & Moldagem B \\
\cline { 2 - 7 } & (Agregado I) & (Agregado I) & (Agregado II) & (Agregado III) & (Agregado I) & (Agregado I) \\
\cline { 2 - 7 } Dias & $\mathrm{f}_{\mathrm{cm}}(\mathrm{MPa})$ & $\mathrm{f}_{\mathrm{cm}}(\mathrm{MPa})$ & $\mathrm{f}_{\mathrm{cm}}(\mathrm{MPa})$ & $\mathrm{f}_{\mathrm{cm}}(\mathrm{MPa})$ & $\mathrm{f}_{\mathrm{cm}}(\mathrm{MPa})$ & $\mathrm{f}_{\mathrm{cm}}(\mathrm{MPa})$ \\
\hline 1 & $20,49 \pm 0,91$ & $20,65 \pm 0,50$ & $16,56 \pm 0,34$ & $19,71 \pm 1,96$ & $19,74 \pm 0,92$ & $18,05 \pm 0,63$ \\
3 & $30,29 \pm 0,85$ & $29,36 \pm 0,28$ & $31,73 \pm 1,65$ & $30,44 \pm 0,94$ & $30,53 \pm 0,96$ & $27,91 \pm 0,80$ \\
7 & $39,22 \pm 0,81$ & $35,85 \pm 1,16$ & $40,03 \pm 0,43$ & $43,83 \pm 0,70$ & $33,85 \pm 1,25$ & $31,88 \pm 1,18$ \\
28 & $44,79 \pm 1,09$ & $45,21 \pm 1,12$ & $43,65 \pm 2,55$ & $46,61 \pm 1,16$ & $40,02 \pm 1,88$ & $36,71 \pm 0,64$ \\
\hline
\end{tabular}

A partir dos valores de tensão e deformação específica obtidos nos ensaios, procedeu-se à determinação do módulo de elasticidade tangente inicial, $\mathrm{E}_{\mathrm{ci}}$, para cada um dos corpos de prova, com base na NBR 8522 [12], considerando o módulo elástico cordal entre 0,5 MPa e 0,3f , conforme a Equação 1.

$$
\mathrm{E}_{\mathrm{ci}}=\frac{\sigma_{\mathrm{b}}-0,5}{\varepsilon_{\mathrm{b}}-\varepsilon_{\mathrm{a}}}
$$

Com: $\sigma_{\mathrm{b}}=$ tensão de ensaio, correspondente a $30 \%$ de $\mathrm{f}_{\mathrm{c}} ; \varepsilon_{\mathrm{a}}=$ deformação específica correspondente a $\sigma_{\mathrm{a}}$ igual a $0,5 \mathrm{MPa} ; \varepsilon_{\mathrm{b}}=$ deformação específica correspondente $\mathrm{a} \sigma_{\mathrm{b}}$.

A Tabela 8 resume os valores médios experimentais obtidos para o módulo estático de elasticidade da argamassa de cimento Portland submetida à compressão simples, $\mathrm{E}_{\mathrm{cm}}$, para cada variável estudada, incluindo o desvio padrão. Para o agregado III o módulo de elasticidade for determinado apenas aos 28 dias.

Tabela 8: Valores médios para o módulo estático de elasticidade da argamassa de cimento Portland.

\begin{tabular}{lllllll}
\hline & \multicolumn{4}{c}{ Fabricante 1 } & \multicolumn{2}{c}{ Fabricante 2 } \\
\cline { 2 - 7 } & Moldagem A & Moldagem B & Moldagem A & Moldagem A & Moldagem A & Moldagem B \\
\cline { 2 - 7 } & (Agregado I) & (Agregado I) & (Agregado II) & (Agregado III) & (Agregado I) & (Agregado I) \\
\cline { 2 - 7 } Dias & $\mathrm{E}_{\mathrm{cm}}(\mathrm{GPa})$ & $\mathrm{E}_{\mathrm{cm}}(\mathrm{GPa})$ & $\mathrm{E}_{\mathrm{cm}}(\mathrm{GPa})$ & $\mathrm{E}_{\mathrm{cm}}(\mathrm{GPa})$ & $\mathrm{E}_{\mathrm{cm}}(\mathrm{GPa})$ & $\mathrm{E}_{\mathrm{cm}}(\mathrm{GPa})$ \\
\hline 1 & $20,98 \pm 1,27$ & $23,68 \pm 0,57$ & $19,35 \pm 2,19$ & $\mathrm{nd}^{*}$ & $22,33 \pm 0,54$ & $19,63 \pm 0,33$ \\
3 & $28,23 \pm 1,63$ & $25,66 \pm 1,70$ & $28,51 \pm 1,41$ & $\mathrm{nd}^{*}$ & $25,35 \pm 2,80$ & $26,55 \pm 1,57$ \\
7 & $30,36 \pm 0,42$ & $27,68 \pm 2,40$ & $29,29 \pm 1,50$ & $\mathrm{nd}^{*}$ & $26,25 \pm 0,81$ & $28,06 \pm 1,74$ \\
28 & $33,73 \pm 0,62$ & $31,16 \pm 0,12$ & $30,25 \pm 3,60$ & $28,79 \pm 2,51$ & $30,42 \pm 1,35$ & $30,84 \pm 1,77$ \\
\hline
\end{tabular}

* não determinado por falha do equipamento de ensaio

As Figuras 1, 2 e 3 mostram os resultados obtidos experimentalmente para a resistência à compressão simples do cimento Portland, $\mathrm{f}_{\mathrm{c}}$, calculada com a média dos resultados obtidos nos ensaios, assim como os valores mínimos exigidos pela NBR 5733 [8], para as idades de 1, 3 e 7 dias, respectivamente, de 14 MPa, $24 \mathrm{MPa}$ e $34 \mathrm{MPa}$. 
Fabricante 1

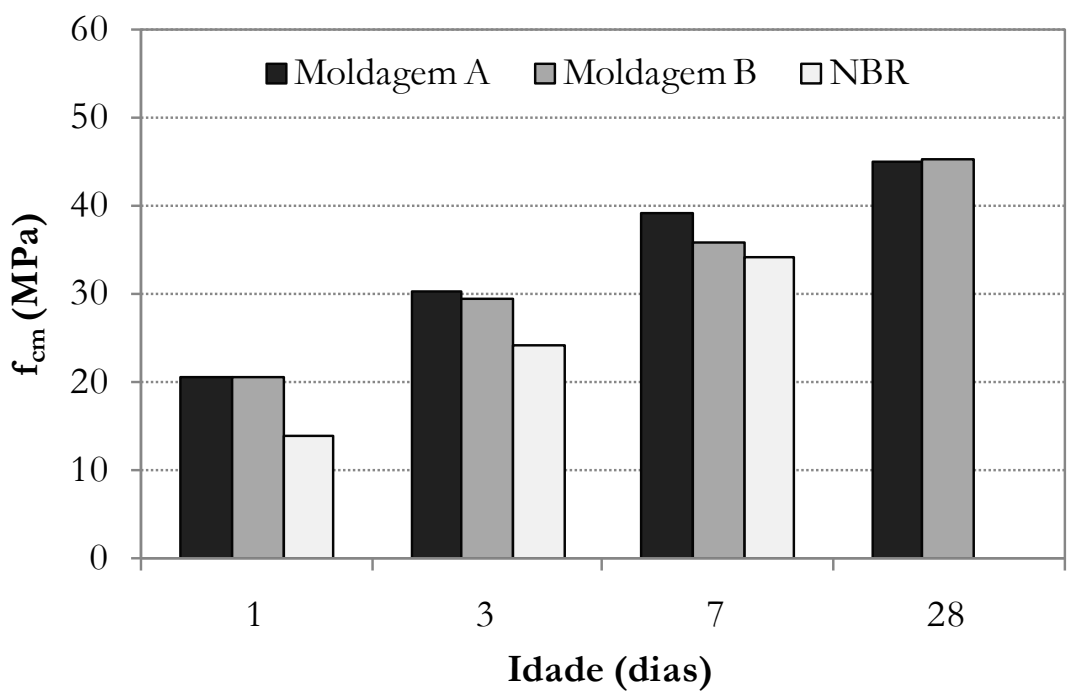

Figura 1: Resistência à compressão do cimento do fabricante 1 para as moldagens A e B.

Fabricante 2

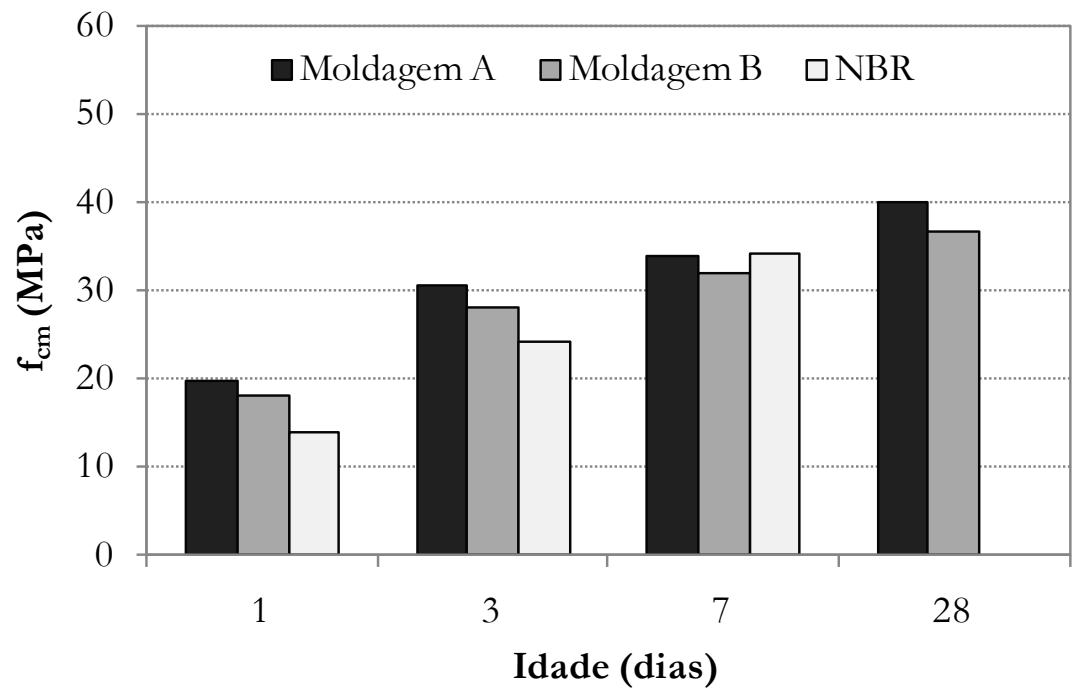

Figura 2: Resistência à compressão do cimento do fabricante 2 para as moldagens A e B. 
Fabricante 1/ Moldagem A

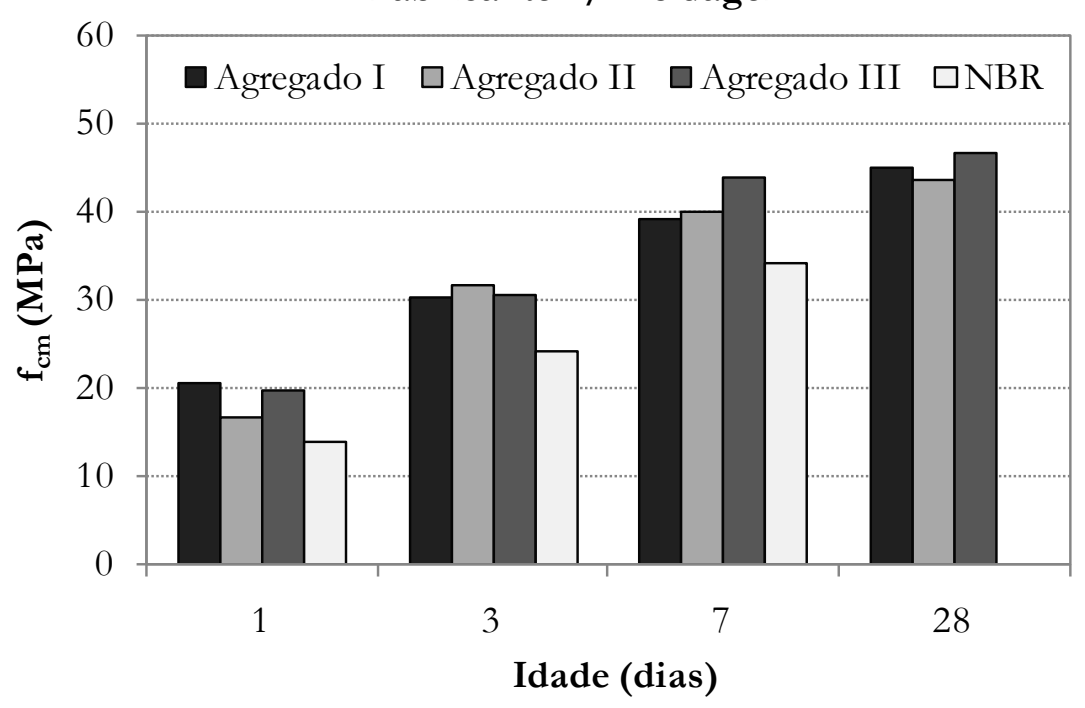

Figura 3: Resistência à compressão do cimento do fabricante 1, moldagem A, para os agregados I, II e III.

É possível observar na Figura 2 que, apesar de se tratar de dois cimentos CP V-ARI do mesmo tipo, o cimento do fabricante 2 não atingiu a resistência à compressão mínima exigida pela norma para a idade de 7 dias, porém nos casos restantes os valores de $\mathrm{f}_{\mathrm{cj}}$ estão acima dos valores mínimos exigidos pela norma.

A fim de avaliar a significância das variáveis propostas no estudo, foi realizada uma análise de variância entre grupos (ANOVA) e para completar a análise ANOVA utilizou-se o teste de Tukey para verificar diferenças entre médias aos pares, ou seja, verificar o $p$-value entre as médias em pares de todos os resultados obtidos, ambas as analises estatísticas foram realizadas no software STATISTIC 7.0. Considerou-se a relação entre variáveis como sendo significativa estatisticamente para valores de $p$-value inferiores a $0,05(\mathrm{Pr}>\mathrm{Fc}=$ $0,05)$.

Primeiramente analisou-se a influência da combinação moldagem $\mathrm{x}$ fabricante $\mathrm{x}$ idade de ruptura na variável resposta resistência à compressão. Mediante a análise estatística da resistência à compressão, mostrada na Tabela 9, verificou-se, como seria de esperar, que as variáveis combinadas tiveram influência significativa na resistência à compressão simples do cimento Portland, com p-value igual a 0,02210. A análise estatística mostra que de forma geral, o cimento do fabricante 1 obteve melhor desempenho na resistência à compressão simples, em parte, por ter maior superfície específica que o cimento do fabricante 2.

Tabela 9: ANOVA aplicada nos resultados dos ensaios de resistência à compressão (Idade x Fabricante x Moldagem).

\begin{tabular}{cccccc}
\hline Variáveis & $\begin{array}{c}\text { Graus de } \\
\text { Liberdade }\end{array}$ & $\begin{array}{c}\text { Soma dos } \\
\text { Quadrados }\end{array}$ & $\begin{array}{c}\text { Médias } \\
\text { Quadradas }\end{array}$ & Fc & Pr>Fc \\
\hline Idade (1) & 3 & 3933,82 & 1311,27 & 22422,35 & 0,00000 \\
\hline Fabricante (2) & 1 & 178,65 & 178,65 & 511,31 & 0,00000 \\
\hline Moldagem (3) & 1 & 46,03 & 46,03 & 69,66 & 0,00009 \\
\hline$(1) *(2)$ & 3 & 102,90 & 34,30 & 17,95 & 0,00000 \\
\hline$(1)^{*}(3)$ & 3 & 23,68 & 7,89 & 13,37 & 0,03476 \\
\hline$(2) *(3)$ & 1 & 5,33 & 5,33 & 3,08 & 0,15498 \\
\hline$(1) *(2) *(3)$ & 3 & 26,66 & 8,89 & 2,08 & 0,02210 \\
\hline Erro & 56 & 143,61 & 2,56 & - & - \\
\hline
\end{tabular}

A Figura 4 mostra que os resultados com 1 e 3 dias foram similares estatisticamente para os dois procedimentos de moldagem (A e B) e fabricantes (1 e 2) com p-value médio de 0,96 com 1 dia e 0,86 aos 3 dias. Aos 7 dias apenas o resultado com cimento de fabricante 1 e moldagem A foi superior aos demais valores, os resultados restantes foram similares estatisticamente com $p$-value médio de 0,71 . Aos 28 dias os resultados foram similares estatisticamente entre os procedimentos de moldagem, porém entre fabricantes os resultados foram distintos, a análise estatística mostrou que os resultados do fabricante 1 foram superiores com $p$ - 
value $<0,05$. De maneira geral, isolando a variável moldagem, nas amostras moldadas com o agregado padronizado, é possível constatar que o procedimento de mistura não influenciou a resistência à compressão simples da argamassa de cimento Portland, ou seja, na maioria dos casos os valores foram estatisticamente similares.

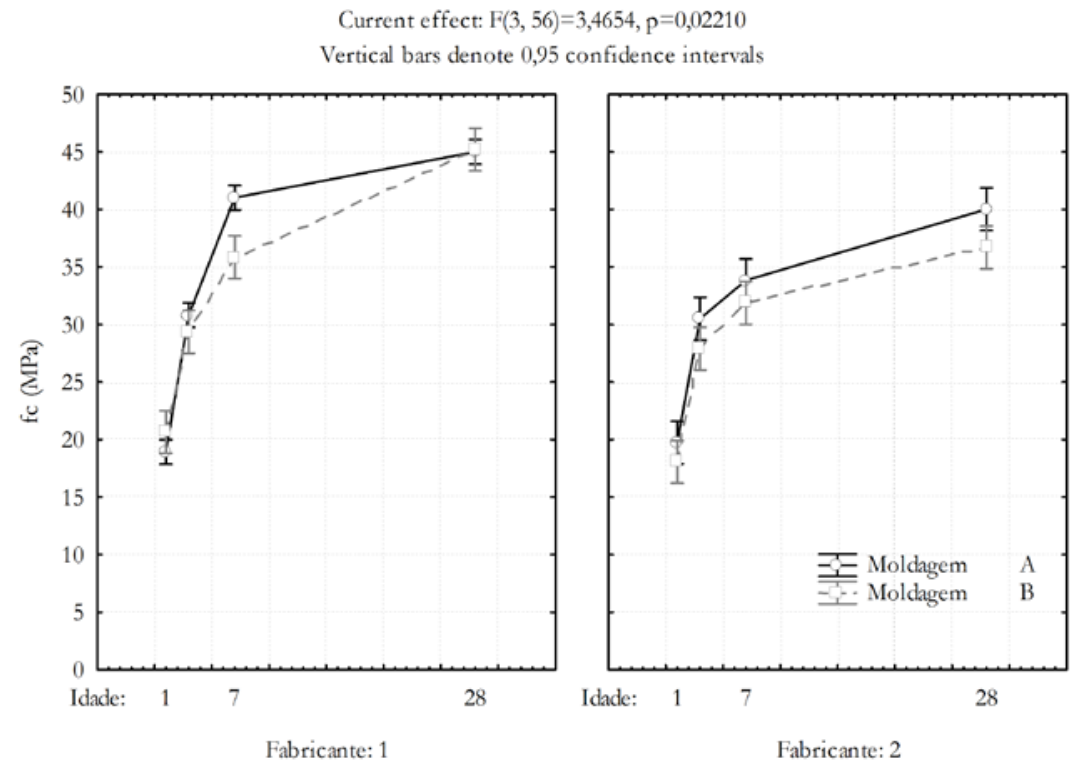

Figura 4: Influência da moldagem na resistência à compressão das argamassas evidenciando os fabricantes 1 e 2.

De forma análoga à resistência, analisou-se a influência da moldagem x fabricante x idade de ruptura na variável resposta módulo estático de elasticidade. Por meio da análise estatística, mostrada na Tabela 10, verificou-se que, quando analisadas conjuntamente, as variáveis apresentaram influência significativa nos resultados experimentais, com $p$-value igual a 0,01221 .

Tabela 10: ANOVA aplicada nos resultados de módulo estático de elasticidade (Idade x Fabricante x Moldagem).

\begin{tabular}{cccccc}
\hline Variáveis & $\begin{array}{c}\text { Graus de } \\
\text { Liberdade }\end{array}$ & $\begin{array}{c}\text { Soma dos } \\
\text { Quadrados }\end{array}$ & $\begin{array}{c}\text { Médias } \\
\text { Quadradas }\end{array}$ & Fc & Pr $>$ Fc \\
\hline Idade (1) & 3 & 712,64 & 237,55 & 45,826 & 0,00000 \\
\hline Fabricante (2) & 1 & 3,14 & 3,14 & 0,605 & 0,43990 \\
\hline Moldagem (3) & 1 & 3,47 & 3,47 & 0,670 & 0,41645 \\
\hline$(1) *(2)$ & 3 & 1,85 & 0,62 & 0,119 & 0,94857 \\
\hline$(1) *(3)$ & 3 & 1,84 & 0,61 & 0,118 & 0,94889 \\
\hline$(2) *(3)$ & 1 & 1,35 & 1,35 & 0,260 & 0,61184 \\
\hline$(1) *(2) *(3)$ & 3 & 61,86 & 20,62 & 3,978 & 0,01221 \\
\hline Erro & 56 & 290,29 & 5,18 & - & - \\
\hline
\end{tabular}

Na Figura 5 é possível observar que, isolando a variável moldagem e fabricante nas amostras com areia padrão, o procedimento de mistura não influenciou significativamente o módulo de elasticidade em todas as idades ensaiadas, ou seja, analisando por idade, isoladamente, o módulo de elasticidade foi similar em todas as idades com p-value $>0,05$. 


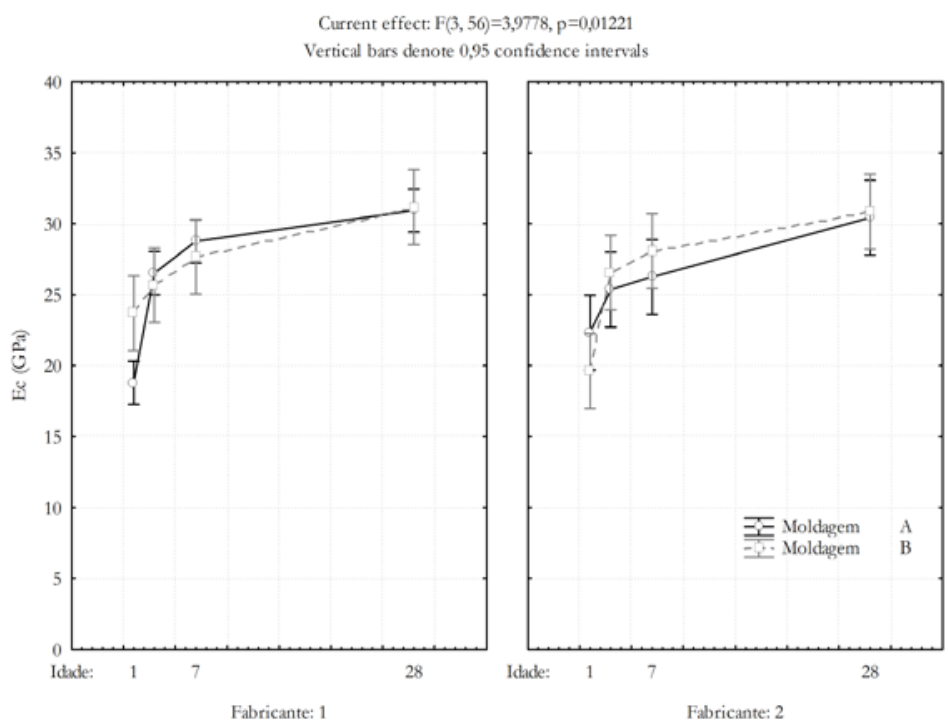

Figura 5: Influência da moldagem e fabricante no módulo de elasticidade das argamassas por idade.

Analisou-se, também, a influência do Agregado (I, II e III), por idade de ruptura (1, 3, 7 e 28 dias), na variável resposta resistência à compressão simples (Tabela 11). Para não alargar excessivamente o programa experimental, a comparação dos resultados para os 3 tipos de agregado miúdo se embasou na combinação padrão de ensaio (Moldagem A, Fabricante 1 e Agregado I), variando apenas o tipo de agregado utilizado nas misturas. Na Figura 6 é possível observar que a variável Agregado teve influência significativa na variável resposta resistência à compressão do cimento, com p-value igual a 0,0015. Por outro lado, analisando isoladamente, foi possível observar que as resistências obtidas para o agregado III foram, de forma geral, superiores às resistências obtidas para os outros agregados. Isto se deve, em parte, ao melhor empacotamento das partículas, consequência do proporcionamento da areia natural em frações padronizadas.

Tabela 11: ANOVA aplicada nos resultados dos ensaios de resistência à compressão (Idade x Agregado).

\begin{tabular}{cccccc}
\hline Variáveis & $\begin{array}{c}\text { Graus de } \\
\text { Liberdade }\end{array}$ & $\begin{array}{c}\text { Soma dos } \\
\text { Quadrados }\end{array}$ & $\begin{array}{c}\text { Médias } \\
\text { Quadradas }\end{array}$ & Fc & Pr>Fc \\
\hline Idade (1) & 3 & 3673,30 & 1224,43 & 755,41 & 0,00000 \\
\hline Agregado (2) & 2 & 28,95 & 14,47 & 8,93 & 0,00126 \\
\hline$(1) *(2)$ & 6 & 50,53 & 8,42 & 5,20 & 0,00150 \\
\hline Erro & 24 & 38,90 & 1,62 & - & - \\
\hline
\end{tabular}

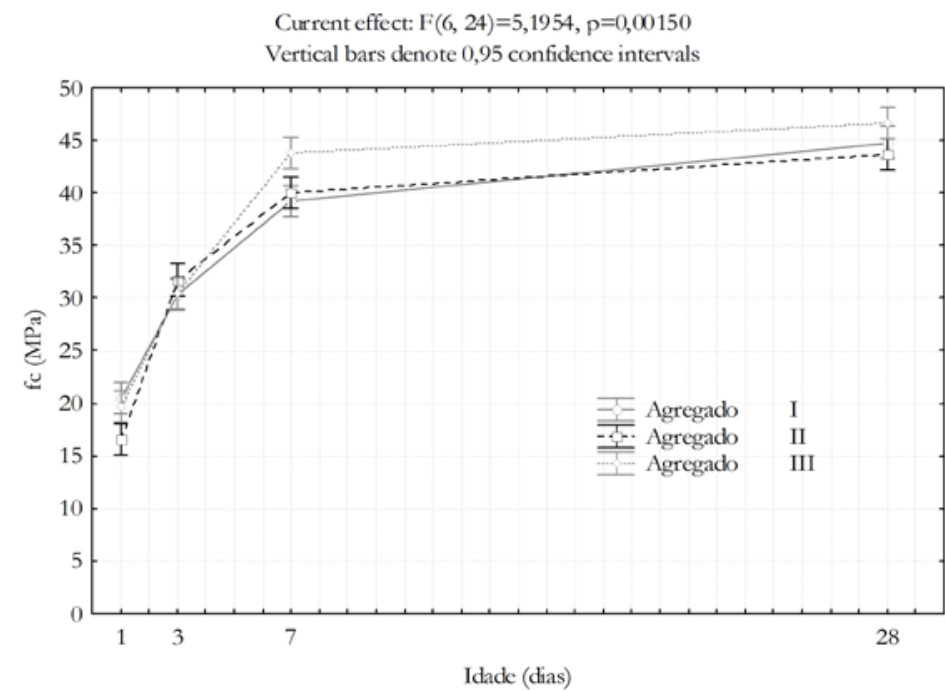

Figura 6: Influência do agregado na resistência à compressão das argamassas por idade. 
Por último, analisou-se a influência do Agregado (I, II, III), por idade de ruptura (1, 3, 7 e 28 dias), na variável resposta módulo estático de elasticidade (Tabela 12). Devido à falha no equipamento de ensaio, para o agregado III só foi possível obter os valores do módulo de elasticidade aos 28 dias de idade. Na Figura 7 observa-se que o tipo de agregado miúdo utilizado na mistura de argamassa não teve influência significativa na variável resposta módulo estático de elasticidade, com o valor $p$-value de 0,41951. O teste de Tukey mostrou que os resultados de módulo de elasticidade foram semelhantes entre si em todas as idades com $p$ value $>0,05$

Tabela 12: ANOVA aplicada nos resultados nos resultados de módulo estático de elasticidade (Idade x Agregado).

\begin{tabular}{cccccc}
\hline Variáveis & $\begin{array}{c}\text { Graus de } \\
\text { Liberdade }\end{array}$ & $\begin{array}{c}\text { Soma dos } \\
\text { Quadrados }\end{array}$ & $\begin{array}{c}\text { Médias } \\
\text { Quadradas }\end{array}$ & Fc & Pr $>$ Fc \\
\hline Idade (1) & - & - & - & - & - \\
\hline Agregado (2) & 1 & 13,05254 & 13,05254 & 3,542017 & 0,07610 \\
\hline$(1)^{*}(2)$ & 3 & 10,95161 & 3,65054 & 0,990632 & 0,41951 \\
\hline Erro & 18 & 66,33103 & 3,68506 & - & - \\
\hline
\end{tabular}

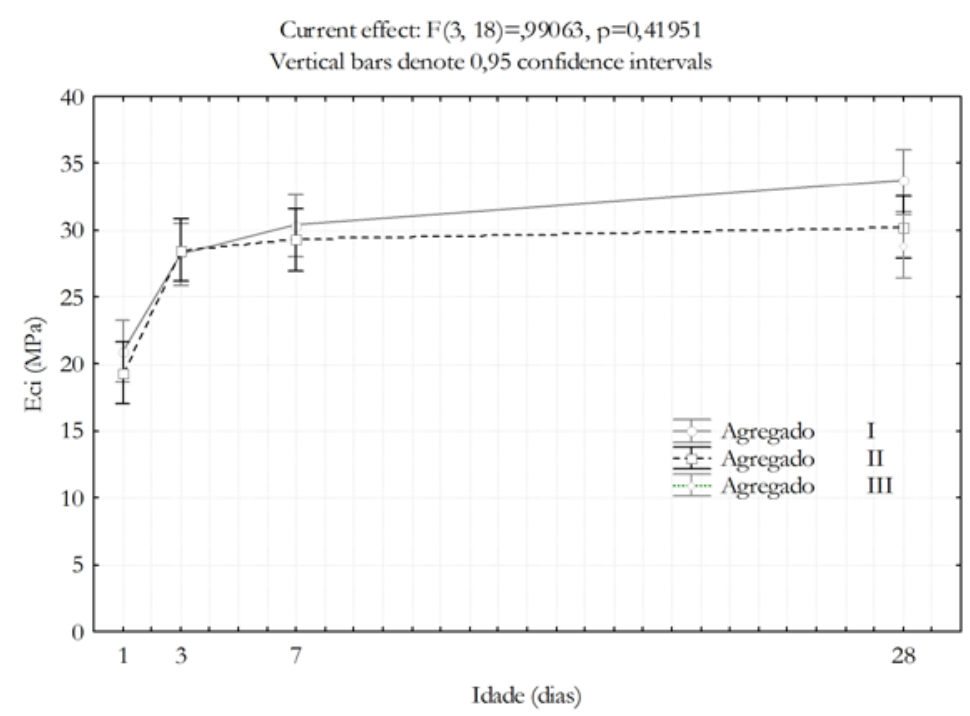

Figura 7: Influência do agregado no módulo de elasticidade das argamassas por idade.

A Figura 8 apresenta os diagramas tensão - deformação específica obtidos nos ensaios de compressão, aos 7 e 28 dias, para a argamassa padrão (cimento do fabricante 1, areia padronizada e misturada de acordo com os procedimentos da norma). A configuração das curvas obtidas em todos os ensaios foi idêntica. É possível observar que a configuração das curvas tensão - deformação específica que foram obtidas experimentalmente para a argamassa se assemelha à configuração típica da curva tensão - deformação específica de um concreto convencional submetido à compressão simples, onde se considera linear a relação entre tensões e deformações específicas do material em um determinado intervalo de valores que, de acordo com a NBR 8522 [12], é válido até $30 \%$ de $\mathrm{f}_{\mathrm{c}}$. 

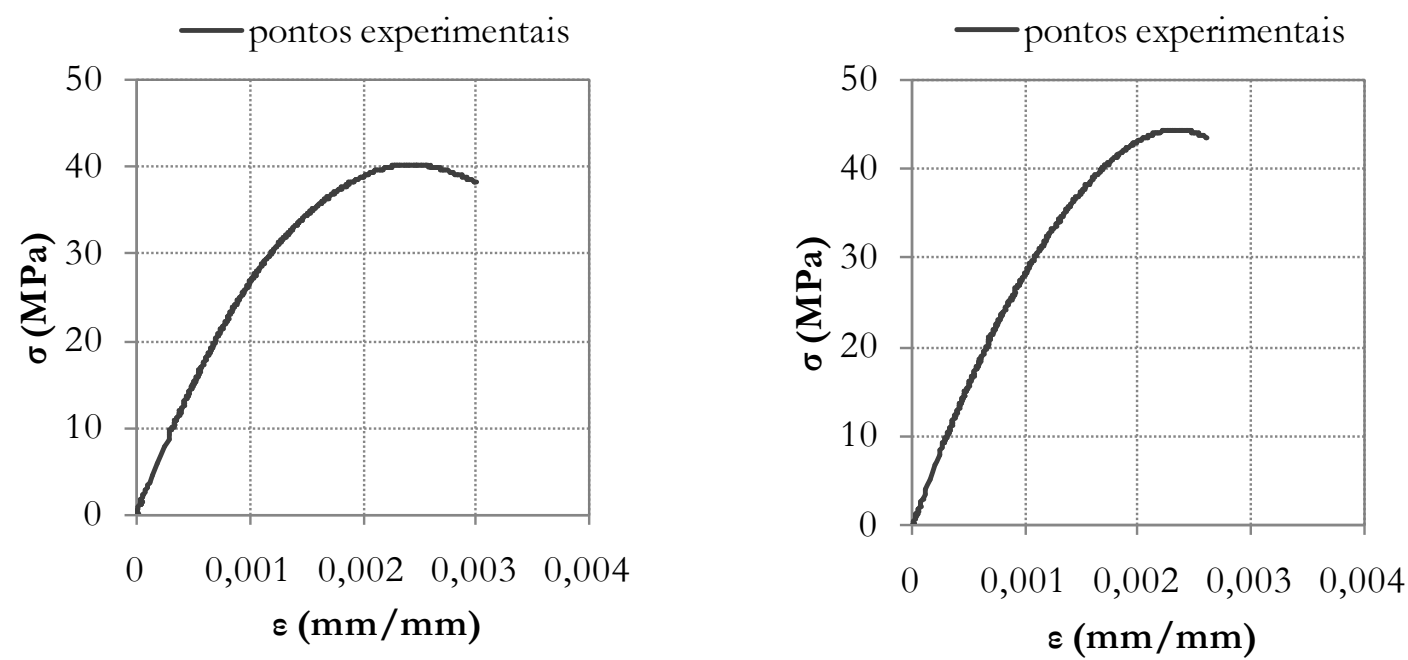

Figura 8: Diagrama $\sigma-\varepsilon$ da argamassa de cimento padrão de ensaio aos (a) 7 dias e (b) 28 dias.

Complementarmente, procurou-se avaliar o grau de correlação entre os modelos teóricos da NBR 6118 [10] e do CEB 90 [11], utilizados para calcular o módulo de elasticidade com base na resistência à compressão, e os resultados obtidos experimentalmente. Foram utilizadas as normas de concreto já que não existe norma específica para o cálculo teórico do módulo de elasticidade de argamassas. A NBR 8522 [12] prescreve que a fórmula para estimar o módulo estático de elasticidade à compressão, $\mathrm{E}_{\mathrm{c}}$, deve-se ser aplicada aos 28 dias de idade para concretos com $\mathrm{f}_{\mathrm{ck}} \leq 50 \mathrm{MPa}$. A NBR 6118 [10] especifica, ainda, que o módulo de elasticidade numa idade $\mathrm{j} \geq 7$ dias pode também ser avaliado através das mesmas expressões substituindose $f_{\text {ck }}$ por $f_{\text {ckj }}$. Observou-se que, realizando o ajuste com todos os pontos experimentais, os coeficientes de determinação foram satisfatórios, obtendo-se $\mathrm{R}^{2}=0,846$ e $\mathrm{R}^{2}=0,761$, respectivamente, para o modelo teórico da NBR 6118 [10] e do CEB 90 [11]. Para melhor visualizar o ajuste dos modelos teóricos aos resultados experimentais o eixo das abscissas foi alterado, de acordo com a Figura 9.
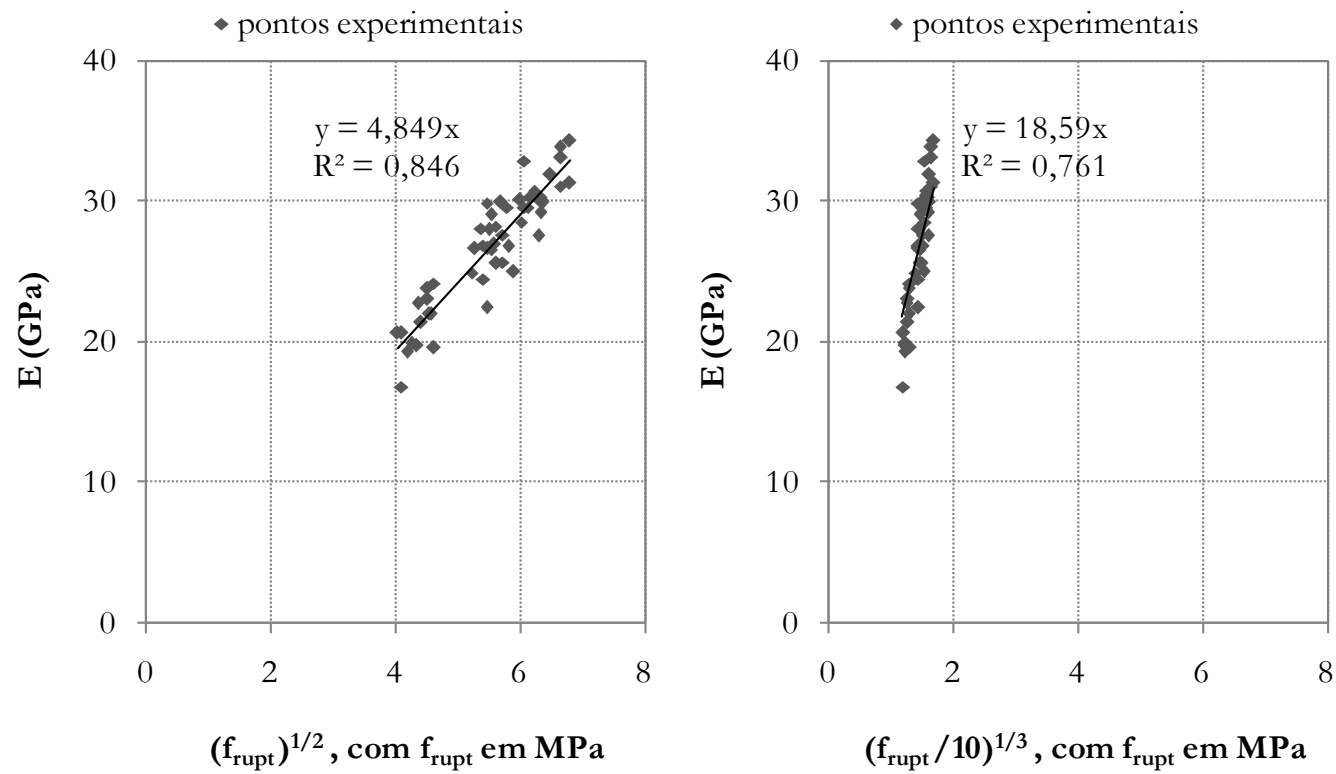

Figura 9: Ajuste do módulo de elasticidade teórico aos resultados experimentais, independentes da idade, com base (a) na NBR 6118 e (b) no CEB 90.

\section{CONCLUSÕES}

Após a análise dos resultados, para as condições prescritas nesta pesquisa, é possível concluir que:

a) A moldagem simplificada, utilizada na preparação das amostras com areia padrão para ensaio, não influenciou significativamente a resistência à compressão simples nem o módulo estático de elasticidade do cimento Portland na maioria dos casos. 
b) O tipo de agregado miúdo utilizado teve influência significativa na resistência à compressão do cimento Portland, mas não impactou os resultados do módulo de elasticidade; por outro lado, foi possível observar que as resistências obtidas para o agregado preparado em laboratório foram, de forma geral, superiores às resistências obtidas para os agregados padrão e natural. Isto se deve, em parte, ao melhor empacotamento das partículas, consequência do proporcionamento da areia natural em frações padronizadas de acordo com a norma.

c) O cimento com maior superfície específica teve melhor desempenho mecânico, expresso pela maior resistência à compressão simples.

d) A relação tensão - deformação específica observada nos ensaios indica que, dentro do âmbito deste trabalho, os procedimentos recomendados pela NBR 8522 [12] foram adequados para a determinação experimental do módulo estático de elasticidade à compressão da argamassa de cimento Portland; por outro lado, a melhor correlação entre o módulo de elasticidade experimental e o módulo de elasticidade teórico foi obtida através das expressões da NBR 6118 [10].

De forma geral, os resultados obtidos destacam que determinadas variações nos procedimentos padrão de ensaio podem alterar significativamente as propriedades mecânicas avaliadas. Por outro lado, algumas simplificações nas metodologias de ensaio poderão constituir alternativas a determinados procedimentos padrão, mais rigorosos e difíceis de colocar em prática.

\section{AGRADECIMENTOS}

Ao CNPq (Conselho Nacional de Desenvolvimento Científico e Tecnológico).

\section{BIBLIOGRAFIA}

[1] ASSOCIAÇÃO BRASILEIRA DE NORMAS TÉCNICAS. NBR 7215: Cimento Portland - Resistência à compressão do Cimento Portland. Rio de Janeiro: ABNT, 1997.

[2] NEVILlE, M. A. Propriedades do Concreto, Tradução Eng. ${ }^{\circ}$ Salvador E. Giammusso, $2^{\text {a }}$ edição rev. atual, Ed. Pini, São Paulo, 1997.

[3] MEHTA, P. K., MONTEIRO, P. J. M. Concreto - Microestrutura, Propriedades e Materiais, $2^{\mathrm{a}}$ edição (Português), Ed. Pini, São Paulo, 2014.

[4] CARNEIRO, A. M. P. Contribuição ao Estudo da Influência do Agregado nas Propriedades de Argamassas Compostas a Partir de Curvas Granulométricas. Tese (Doutorado em Engenharia Civil) - Escola Politécnica da Universidade de São Paulo, São Paulo, SP, 1999.

[5] ARAÚJO, G. S. Estudos de Parâmetros Texturais das Areias para Argamassas de Revestimento através da Análise de Imagens. 104p. Dissertação (Mestrado em Engenharia Civil) - Universidade Federal do Espírito Santo. Vitória, ES. 2001.

[6] SCHUTTER, G., POPPE, A. M. "Quantification of the Water Demand of Sand in Mortar". Construction and Buildings Materials, n. 18, p. 517-521. 2004.

[7] ASSOCIAÇÃO BRASILEIRA DE NORMAS TÉCNICAS. NBR 7214: Areia normal para ensaio de cimento — Especificação. Rio de Janeiro: ABNT, 2012.

[8] ASSOCIAÇÃO BRASILEIRA DE NORMAS TÉCNICAS. NBR 5733: Cimento Portland de alta resistência inicial. Rio de Janeiro: ABNT, 1991.

[9] PACHECO, J., HELENE, P. "Controle da Resistência do Concreto". Asociación Latinoamericana de Control de Calidad, Patología y Recuperación de la Construcción, Boletim Técnico, n. 9, pp. 3-18. Mérida, México, 2013.

[10] ASSOCIAÇÃO BRASILEIRA DE NORMAS TÉCNICAS. NBR 6118: Projeto de Estruturas de Concreto - Procedimentos. Rio de Janeiro: ABNT, 2014.

[11] COMITE EURO-INTERNACIONAL DU BETON. CEB 90: Model Code for Concrete Structures. CEB/FIP, 1990.

[12] ASSOCIAÇÃO BRASILEIRA DE NORMAS TÉCNICAS. NBR 8522: Concreto - Determinação do Módulo Estático de Elasticidade à Compressão. Rio de Janeiro: ABNT, 2008. 